\title{
Immunotherapy for Chronic Hepatitis B: Will This Lead to Rome?
}

\author{
${ }^{1}$ Sheikh Mohammad Fazle Akbar, ${ }^{2}$ Mamun-Al-Mahtab \\ ${ }^{1}$ Department of Medical Sciences, Toshiba General Hospital, Tokyo, Japan \\ ${ }^{2}$ Department of Hepatology, Bangabandhu Sheikh Mujib Medical University, Dhaka, Bangladesh
}

Correspondence: Sheikh Mohammad Fazle Akbar, Principal and Investigator, Department of Medical Sciences, Toshiba General Hospital, Tokyo, Japan, e-mail: sheikh.akbar@po.toshiba.co.jp

\section{ABSTRACT}

The entity and concept of immune therapy against chronic infections and cancers is still elusive. Credible preclinical studies and clinical trials are scare in this field due to improper understating about cellular and molecular mechanism of these diseases. In addition, immune interventional strategies against these diseases have not been properly formulated. A sketch about immune pathogenesis of these diseases and interventional strategies has been formulated in this communication.

Keywords: Immune therapy, Chronic infection, Cancers, Strategy.

\section{INTRODUCTION}

Not only hepatitis B virus (HBV), but also several other viral, bacterial and protozoal infections, and many noninfectious chronic diseases, have effectively been controlled or strategies have been developed to control these diseases in developed and rich countries of the world through implementation of several prophylactic and therapeutic approaches. Although these measures were initiated almost simultaneously in both developed and developing countries, the beneficial effects of the global fighting against microbial agents have not been reproduced or duplicated in developing and resource-constrained regions and countries. ${ }^{1,2}$ However, developing countries have been flooded with mutant microbial agents those are difficult to control and treat.

In the context of HBV infection, new cases of HBV infection are negligible, chronic HBV infection is a treatable and emergence of mutant HBV has effectively been controlled in rich and developed countries during last three decades. On the contrary, about 50 million healthy people are infected by HBV each year in developing countries in spite of availability of potent prophylactic vaccines against $\mathrm{HBV}$. Also, the therapeutic regimen against chronic hepatitis B (CHB) has not been optimized in developing countries that would be able to provide benefit to majority of treated patients and reduces adverse effects. Health care delivery system of developing countries, inadequate resources and defective strategies is partially responsible for these unfortunate situations. ${ }^{3}$ However, elusive regimen of antiviral therapy in CHB, elicit use of drugs and lack development of society-friendly therapeutic approaches are equally accountable for miserable conditions of developing countries to fight $\mathrm{HBV}$.,
Out of 160 million people of Bangladesh, about 50 million or more are supposed to be infected with the $\mathrm{HBV}$ at some point of their life. Also, conservative estimates show that about 8 million people of this country are chronic HBV carrier, a condition that implies that these patients would continue to harbor HBV for the rest of their life even being treated with all types of antiviral drugs. To treat millions of patients with CHB at Bangladesh, antiviral drugs are available in the market. Out of several forms of HBV, available antiviral drugs can only reduce growth of replicating HBV, but these drugs are unable to control ccCDNA, a form of HBV DNA that is integrated in the liver and can act as template for future replication. Be sure that none of these drugs can eliminate HBV completely from any patient with CHB. Immune modulatory capacity of these drugs is not so promising. Thus, these drugs can control liver damage in one-third or lesser numbers of CHB patients for certain duration. Taken together, the role of these drugs in delaying liver cirrhosis and liver cancer is still elusive. ${ }^{6}$ Although these antiviral drugs can induce positive therapeutic outcome in one-third of treated patients, these benefit would come if the following preconditions are fully complied: (1) The patients should be properly assessed by a trained physician about his/her liver disease activity prior to starting antiviral drug therapy, (2) the patients should take drug on a regular basis for prolonged period or even for years or for life, (3) the patient should be properly followed up and several virological, biochemical and immunological parameters of liver function tests should be done periodically, and (4) the patient should be checked for mutant HBV and flare of liver diseases regularly. It is natural to ask how many CHB patient of Bangladesh can 
fully adhere to these preconditions and how many CHB patients are informed of scopes and limitations of antiviral drug therapy by attending physicians. Out of about 2 million treatable patients of CHB in Bangladesh, these conditions may be addressed by few thousands patients of big cities of Bangladesh. However, if treatment of CHB patients is done with antiviral drugs without adhering to these conditions, the patients will develop several complications of HBV infection that would compromise his/her quality of life tremendously and the country will be filled up with mutant HBV. Control of mutant HBV may not accomplished by health care delivery system of Bangladesh even with global support.

Dissecting the present situations, we found that: (1) Present regimen of antiviral therapy is not applicable in its present form in Bangladesh and most other developing countries, and (2) the surrogate markers of antiviral therapy in CHB patients that have been shown by several eminent scholars may be misleading or untrue. ${ }^{7}$ Based on evidences, a new approach of therapeutic maneuvers for CHB patients has been developed that seems to be: (1) Safe in short and long perspective, (2) cheap for patients of developing countries, (3) moderately effective, (4) devoid of inducing mutant HBV strains and (5) adjusted with the socioeconomic and cultural behaviors of developing countries. The principle of immune therapy of CHB patients is based on a concept of 'paradise lost' to 'paradise gain' phenomenon. About $100 \%$ patients with acute HBV infection and $80 \%$ of chronic HBV infection effectively control HBV replication and liver damages by HBsAg and HBcAgspecific immunity. However, patients with CHB with progressive liver damages are unable to inducing and maintaining protective immunity efficiently. To establish an antigen-specific immune therapy in CHB patients, we have provided evidences: (1) Countering self/nonself theory of immunity and (2) discarding neonatal tolerance theory. HBV-based vaccine has been applied in an animal model of HBV-carrier stage, HBV-transgenic mice, for about two decades to assess: (1) Safety, (2) immunogenecity and (3) antiviral and liver supporting potentiality using several adjuvants and therapeutic designs. During last one decade, we have reproduced some of our studies of HBV-transgenic mice in patients with $\mathrm{CHB}$, an approach to bring information of the laboratory benches to patient's bedsides. ${ }^{8}$ Finally, during last 3 years, we have accomplished a phase I/II clinical trial in CHB patients in which $\mathrm{HBsAg} / \mathrm{HBcAg}$ combined vaccine was given to CHB patient by nasal as well as by parental route. ${ }^{9}$ Two years after therapy termination, all patients are safe and none have developed mutant HBV. The vaccine reduced HBV DNA in majority of patients and HBV DNA negativity was detected in about 40 to 50\% CHB patients. Most of the patients have been showing a normal alanine aminotransferase (ALT) level, a fact that imply that liver damages have effectively been controlled. A phase III clinical trial has been planned with this vaccine in CHB patients. Although more studies are needed to optimize immune therapy in CHB patients, our concept of immune therapy may also be applied to treat hepatitis C, malaria, tuberculosis, HIV, autoimmune diseases, allergic conditions and all sorts of cancers. What we need is the development of appropriate viral, bacterial, fungal or cancer antigen(s) and a group of people who assume that 'we should follow the road that should be followed, but not one that have been followed by our traditional predecessors'. If people of allied fields of developing countries become interested to develop these therapeutic approaches, therapy with low side effects and moderate efficacies can be developed for combating microbial infections and managing cancers in developing and resource-constrained countries. Basically, these therapeutic regimens act on restoration of one's selfresistance to fight pathological diseases, a philosophy that has been unfolded in this subcontinent and ancient Chinese, Egyptian and Greece civilizations, but has not been properly optimized and updated for medical science of 21st century.

\section{REFERENCES}

1. Zanetti AR, Zappa A. Emerging and re-emerging infections at the turn of the millennium. Haemophilia 2010;16(S1):7-12.

2. Samb B, Desai N, Nishtar S, et al. Prevention and management of chronic disease: A litmus test for health-systems strengthening in low-income and middle-income countries. Lancet 2010; 20;376:1785-97

3. Liaw YF. Antiviral therapy of chronic hepatitis B: Opportunities and challenges in Asia. J Hepatol 2009;51:403-07.

4. Akbar SM, Hiasa Y, Mishiro S, Onji M. Treatment of hepatitis B virus-infected patients: Utility of therapeutic recommendations in developing countries. Expert Opin Pharmacother 2009;10:1605-14.

5. Akbar SM, Al-Mahtab M, Hiasa Y. Future aspects of therapy for hepatitis B virus infection: Value of surrogate markers, innovative therapy, and global collaboration. J Gastroenterol (in press).

6. Wilt TJ, Shamliyan T, Shaukat A, Taylor BC, MacDonald R, Yuan JM, et al. Management of chronic hepatitis B. Evid Rep Technol Assess (Full Rep) 2008;174:1-671.

7. Shamliyan TA, MacDonald R, Shaukat A, Taylor BC, Yuan JM, Johnson JR, et al. Antiviral therapy for adults with chronic hepatitis B: A systematic review for a National Institutes of Health Consensus Development Conference. Ann Intern Med 2009;150:111-24

8. Akbar SM, Yoshida O, Abe M, Hiasa Y, Onji M. Engineering immune therapy against hepatitis B virus: Hepatol Res 2007;37(S):S351-56.

9. Mahtab MA, Akbar SM, Rahman S, Rubido JCA, Mishiro S. Safety, efficacy and immunogenecity of a therapeutic vaccine containing HBsAg and HBcAg by nasal route in patients with chronic hepatitis B. J Hepatol 2010;52:392. 